\title{
A Compact Vibration Reduced Set-up for Scanning nm-XRF and STXM.
}

$\underline{\text { Janin Lubeck }}^{1^{*}}$, Christian Seim $^{1}$, Aurélie Dehlinger $^{2}$, Andreas Haidl $^{3}$, Philipp Hönicke ${ }^{1}$, Yves Kayser $^{1}$, Rainer Unterumsberger ${ }^{1}$, Claudia Fleischmann ${ }^{4}$ and Burkhard Beckhoff ${ }^{1}$

${ }^{1 .}$ Physikalisch-Technische Bundesanstalt, X-ray Spectrometry, Abbestr. 2-12, 10587 Berlin, Germany

2. Technische Universität Berlin, Institute for Optics and Atomic Physics, Hardenbergstr. 36, 10623

Berlin, Germany

${ }^{3 .}$ University of Applied Science Koblenz - RheinAhr Campus, Institute for X-optics, Joseph-RovanAllee 2, 53424 Remagen, Germany

4. imec, Kapeldreef 75,3001 Leuven, Belgium

* Corresponding author, janin.lubeck@ptb.de

Synchrotron radiation based scanning X-ray spectroscopy (XRS) can determine both elemental concentrations and chemical binding states with high spatial resolution down to the nanoscale. Within the last decade, the need for such high-resolution analytical methods in application fields such as life science, energy storage and conversion as well as climate protection has steadily increased. In order to quantify nanoelectronics, pharmaceuticals, biomolecules and medical imaging contrast agents in biological tissue and cells, the development and adaptation of traceable methodologies are crucial. XRS can be quantitative and recent advances in a novel kind of vibration reduced X-ray fluorescence (XRF) analysis have pushed the spatial resolution towards the $100 \mathrm{~nm}$ regime. This could provide the missing link for quantification across the length scales.

There are only a few scanning X-ray microscopes that offer XRF analysis in combination with scanning transmission X-ray microscopy (STXM) such as Elettra-TwinMic (STXM and XRF); Diamond - I08 (STXM and XRF); PETRA III - P04 AnImaX (STXM and XRF); Advanced Light Source - 11.0.2 (STXM and spectroscopy) and Canadian Light Source - 10ID-1 (STXM and spectroscopy).

With the advent of UHV-compatible piezo stages with nanometer resolution, it is possible to mount optical elements in a compact manner on a single platform. Relevant parts of any external vibrations might be reduced by such a single platform. The lay-out of its stages and related holders is to ensure amplitudes of relative vibrational movements to be smaller than the absolute ones by avoiding any resonance frequencies. We realized this approach in a compact translational and rotational set-up to align beamstops (or diagnostics), zone plates, order sorting apertures (OSA) and samples on a single mounting plate (see fig.1). This plate was mounted on a sample manipulator of non-microscopy equipment such as an XRF instrument in our case [1]. Furthermore, its overall size offers versatile areas of operation as the compact set-up can be readily inserted in a wide range of experimental (vacuum) chambers [2] through their load-locks. Our nm-XRF and STXM set-up is mounted on a 6-axis manipulator [1] which allows for an alignment of all three linear as well as all three rotational axes. The optics and the transmission sample are mounted on piezo positioning stages with 8 axes providing $1 \mathrm{~nm}$ resolution and encoder control on which the zone plate can be aligned in the X-Y plane (perpendicular to the optical axis) and the OSA as well as the transmission sample in X-Y-Z. The fluorescence samples are mounted on a 4-axis manipulator which can be moved in and out of the beam. The optical elements of the scanning X-ray microscope consist of an Au zone plate (with a diameter of $360 \mu \mathrm{m}$, an outer zone width of $25 \mathrm{~nm}$ and an integrated $50 \mu \mathrm{m}$ central stop) and a $50 \mu \mathrm{m}$ OSA. All measurements presented here are carried out with photon energy of $1500 \mathrm{eV}$ at PTB's plane grating monochromator (PGM) 
beamline for undulator radiation [3] at BESSY II. 3 m upstream of the experimental station containing the XRF microscope set-up, pinholes with diameters of $50 \mu \mathrm{m}$ and $100 \mu \mathrm{m}$ can be placed in the beam path at the PGM focal plane to confine and optimize the X-ray beam before it is focused by the zone plate on the sample plane. Between these pinholes and the zone plate as well as behind the sample plane photodiodes can be inserted.

Two objects are used to examine the performance of the novel type of scanning XRF microscope. The test object on the transmission manipulator (see fig.1) is a penetrable Siemens star on a $\mathrm{Si}_{3} \mathrm{~N}_{4} \mathrm{membrane}$ completely covered with a $133 \mathrm{~nm}$ thick layer of $\mathrm{W}$, while for the fluorescence manipulator we chose a silicon wafer with an alternating Ge-Si-wafer bar test structure. Fig. 2 shows an X-Y plot of the transmission signal through a quarter of a Siemens star with the $50 \mu \mathrm{m}$ pinhole inserted in the beam path at the PGM focal plane. The second to smallest structures (approximately $100 \mathrm{~nm}$ ) are clearly resolved and the resolution is comparable to the $\mathrm{X}$ and $\mathrm{Y}$ direction. This was somewhat degraded without any pinhole or the $100 \mu \mathrm{m}$ pinhole inserted where deterioration in the Y direction was observed, which presumably calls for further PGM beamline optimizations towards the resolution otherwise defined by the $25 \mathrm{~nm}$ outer-most zone width. There are several potential reasons why the transmission experiments did not achieve this value. Firstly, at $1500 \mathrm{eV}$ the $133 \mathrm{~nm}$ layer of $\mathrm{W}$ absorbs only $35 \%$ of the incoming $\mathrm{X}$-rays, which leads to lower contrast in comparison to complete absorbance. Since contrast diminishes closer to a microscope's resolution capability limits, which is especially undesirable if the smallest structures are to be resolved. A second explanation could be remaining vibrational contributions to the single mounting stage. This will be further investigated using a slightly modified set-up.

For the XRF experiments the Ge-Si-sample was measured at an angle of incidence of $60^{\circ}$ with an energy-dispersive silicon drift detector (SDD) characterized by PTB. The highest spatial resolution achieved in initial experiments was in the range of $130 \mathrm{~nm}$ to $140 \mathrm{~nm}$ which is in line with the probed sample dimensions. Follow-up experiments are focused at traceable XRS of appropriate and even smaller test structures. Extending the accessible photon energy range of the PGM beamline will enable substantial set-up optimizations with the aim to further verify the capabilities of our vibration reduced approach and its qualification for quantification at the nanoscale.

[1] J. Lubeck et al. Review of Scientific Instruments 84, no. 4 (2013) 045106.

[2] J. Lubeck et al. AIP Conference Proceedings 1741, no. 1 (2016) 030011.

[3] K.J.S. Sawhney et al. Nuclear Instruments and Methods in Physics Research Section A: Accelerators, Spectrometers, Detectors and Associated Equipment 390, no. 3 (1997) 395-402. 


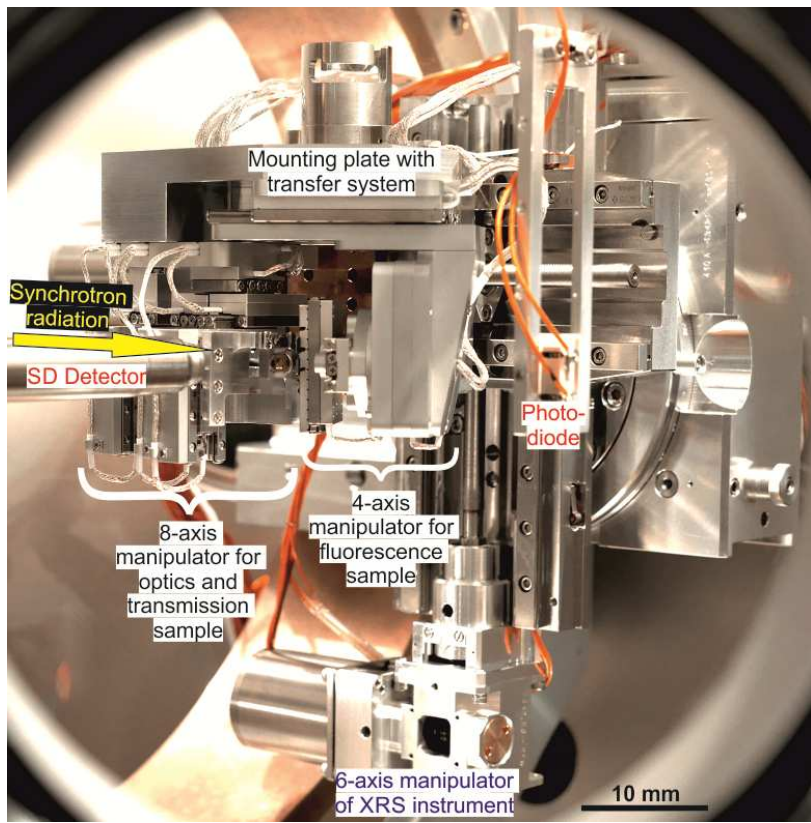

Figure. 1. Compact vibration reduced scanning $\mathrm{X}$-ray fluorescence microscope.

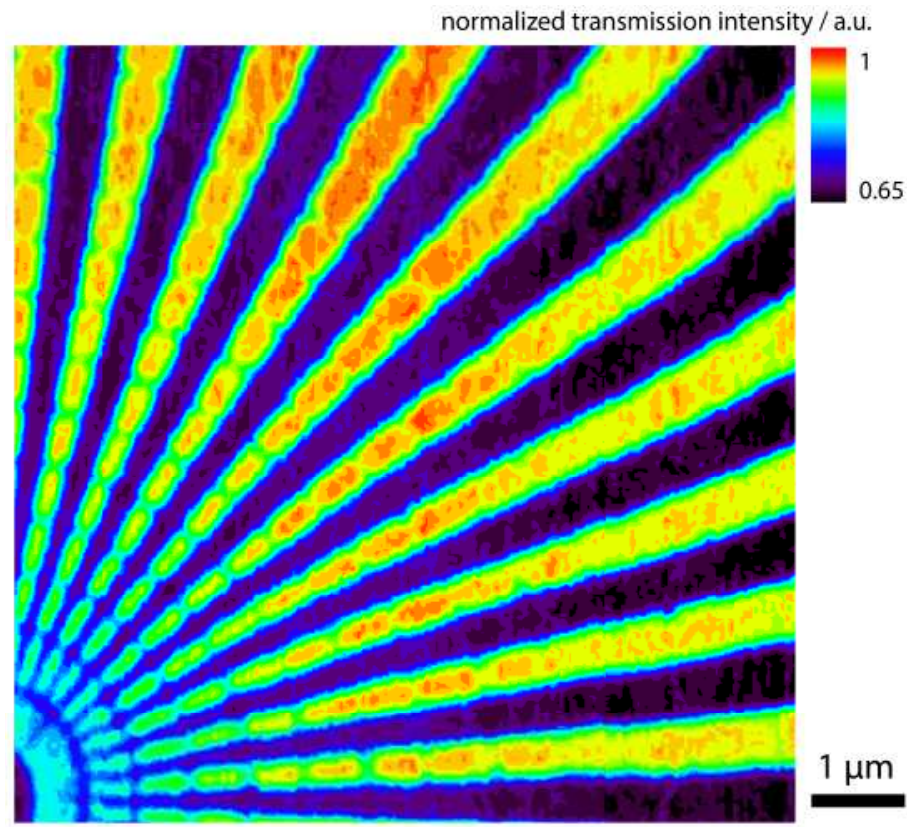

Figure. 2. Transmission map of a Siemens star structure at $1500 \mathrm{eV} .100 \mathrm{~nm}$ structures are clearly resolved. 
https://doi.org/10.1017/S1431927618013181 Published online by Cambridge University Press 